\title{
The association between soluble klotho and cardiovascular parameters in chronic kidney disease: results from the KNOW-CKD study
}

Hyo Jin Kim', Eunjeong Kang ${ }^{2}$, Yun Kyu Oh², Yeong Hoon Kim³ ${ }^{3}$ Seung Hyeok Han ${ }^{4}$, Tae Hyun Yoo ${ }^{4}$, Dong-Wan Chae ${ }^{2}$, Joongyub Lee ${ }^{5}$, Curie $\mathrm{Ahn}^{2}$ and Kook-Hwan $\mathrm{Oh}^{2^{*}}$ (i)

\begin{abstract}
Background: Klotho, a protein linked to aging, has emerged as a pivotal player in mineral bone metabolism and might explain the relationship between chronic kidney disease (CKD) and cardiovascular disease (CVD). The present study aimed to investigate the association between serum klotho and cardiac parameters from a large-scale Korean CKD cohort.

Methods: We analyzed 2101 participants from KoreaN Cohort Study for Outcome in Patients With Chronic Kidney Disease (KNOW-CKD) cohort who had been measured for serum klotho levels. Left ventricular hypertrophy evaluated by left ventricular mass index (LVMI) and arterial stiffness measured by brachial-to-ankle pulse wave velocity (baPM) were explored as cardiovascular parameters.

Results: Patients were $53.6 \pm 12.2$ years old and $61.1 \%$ were male. The mean estimated glomerular filtration rate (eGFR) was $53.0 \pm 30.7 \mathrm{~mL} / \mathrm{min} / 1.73 \mathrm{~m}^{2}$. The median serum klotho level was 536 (interquartile range [IQR]: 420-667) pg/mL. Advanced CKD stages were associated with lower serum klotho levels $(P<0.001$, $P$ for linear trend $<0.001)$. Ascending quartiles of klotho were significantly associated with decreased LMVI $(P<0.001, P$ for linear trend $<0.001)$. A multivariable linear regression model showed serum klotho had a significant inverse association with LVMI $(\beta-0.04 ; 95 \% \mathrm{Cl}$ [confidence interval] $-0.004,-0.00007 ; P=0.041)$. However, there was no significant association between serum klotho and baPW after adjustment ( $\beta 0.003 ; 95 \% \mathrm{Cl}-0.04,0.05 ; P=0.876)$.
\end{abstract}

Trial registration: This trial was registered on ClinicalTrials.gov on 28 June 2012 (NCT01630486).

Conclusions: Serum klotho was an independent biomarker of LVMI, but not arterial stiffness.

Keywords: Serum klotho, Soluble klotho, Chronic kidney disease, Left ventricular mass index, Left ventricular hypertrophy, Pulse wave velocity

\section{Background}

Patients with chronic kidney disease (CKD) present with a higher burden of cardiovascular disease (CVD) and cardiovascular mortality than the general population $[1,2]$. Left ventricular myocardial hypertrophy, the most commonly diagnosed cardiovascular abnormality in CKD patients, is secondary to both volume and pressure overload [3]. Cardiac hypertrophy is an important cause of cardiovascular

\footnotetext{
* Correspondence: khoh@snu.ac.kr

${ }^{2}$ Department of Internal Medicine, Seoul National University College of

Medicine, Seoul, Korea

Full list of author information is available at the end of the article
}

morbidity and mortality for CKD patients because it can lead to congestive heart failure, arrhythmia, ischemic cardiomyopathy, even in the absence of coronary artery disease, and sudden death in CKD patients [4-6]. Arterial stiffness in CKD patients caused by arteriosclerosis with thickening and stiffening of the arterial wall [7] brings about cardiac hypertrophy and a negative prognostic value for CVD $[8,9]$.

Mineral bone metabolism is important in CKD, and progressive deterioration of calcium-phosphorus homeostasis is associated with cardiovascular complications. Impaired calcium-phosphorus homeostasis can cause 
cardiac hypertrophy and vascular calcification. Klotho has emerged as a pivotal player in calcium-phosphorus homeostasis and mineral metabolism regulation in CKD, and it might explain the relationship between CKD and CVD. The Klotho gene, which was originally identified as an aging suppressor gene, is closely associated with CKD. In a previous study, klotho knock-out mice had similarities with CKD patients, such as hyperphosphatemia, ectopic soft tissue calcification, and arteriosclerosis [10], suggesting that CKD might result from a state of klotho deficiency. Thus, in addition to serving as a biomarker for CKD, klotho deficiency is also a pathogenetic indicator for both renal and extra-renal complications in CKD [11]. In previous experimental studies, restoration of serum klotho levels ameliorated cardiac hypertrophy and vascular calcification [12, 13], and haplodeficiency of the Klotho gene caused arterial stiffness [14]. Clinical data supporting the above experimental studies are scarce and present mixed results [15]. Yang et al. [16] showed that cardiac hypertrophy evaluated by left ventricular mass index (LVMI) was negatively associated with serum klotho in 86 CKD patients. They did not show the association between klotho and LVMI for CKD patients in an adjustment model. In another study, there was no significant association between serum klotho and LVMI in dialysis patients [17]. Previous studies have been conducted on a small number of patients and few studies have focused on CKD patients for the association between klotho and LVMI. Given the negative effects of klotho deficiency, its associated cardiovascular complications in preclinical studies, and the limited number of clinical studies, the current study aimed to investigate the association between serum klotho and cardiovascular parameters in CKD patients, using the baseline crosssectional data set of a large-scale Korean CKD cohort.

\section{Methods}

\section{Study population}

The KoreaN Cohort Study for Outcome in Patients With Chronic Kidney Disease (KNOW-CKD) was a Korean multicenter prospective cohort study that enrolled participants with CKD from stage 1 to 5 (predialysis) from nine clinical centers of major university-affiliated hospitals in Korea. Details about the study design and methods are described elsewhere [18]. Subjects with severe heart failure (New York Heart Association Class III or IV) were excluded from enrollment according to study protocol. Among the 2238 participants enrolled in the KNOW-CKD study from June2011 to January2016, 2113 individuals had their serum klotho levels assayed. Twelve patients were excluded as extreme values (serum klotho either lower than detectable range or above $6000 \mathrm{pg} / \mathrm{mL}$ ). These values were not influence value by Cook's distance analysis. Finally 2101individualswere included in the cross-sectional analysis. The study protocol was approved by the ethical committee of each participating clinical center, including the Institutional Review Boards of Seoul National University Hospital, Severance Hospital, Kangbuk Samsung Medical Center, Seoul St. Mary's Hospital, Gil Hospital, Eulji General Hospital, Chonnam National University Hospital, and Busan Paik Hospital. All participating patients provided written informed consent. The study protocol was in accordance with the principles of the Declaration of Helsinki.

\section{Clinical data collection and laboratory analyses}

Baseline demographic characteristics and laboratory values at enrollment were extracted from an electronic data management system (http://www.phactaX.org). Serum creatinine was measured by an isotope dilution mass spectrometry (IDMS)-traceable method [19] at a central laboratory. The estimated glomerular filtration rate (eGFR) was estimated using the Chronic Kidney Disease Epidemiology Collaboration (CKD-EPI) creatinine eq. [20].The serum $\alpha$-klotho level was measured using an enzyme-linked immunosorbent assay (ELISA) kit (Immuno-Biological Laboratories Co., Gunma, Japan) according to the manufacturer's protocol 1 [21]. The intra-assay and inter-assay coefficients of variation were 2.7-3.5\% (Klotho levels186.64-2968.78 pg/mL) and $2.9-11.4 \%$ (Klotho levels $165.47-2903.01 \mathrm{pg} / \mathrm{mL}$ ), respectively. The data for intra-assay and inter-assay coefficients of variations were confirmed in our central laboratory by measurements of a serum control in 20 repeats on each ELISA plate. The intra-assay and inter-assay coefficients of variations were $0.57-1.78 \%$ and $3.01-6.12 \%$ (Klotho levels $120.30-4468.50 \mathrm{pg} / \mathrm{mL}$ ), respectively. The standard curve was found to be linear up to $4000 \mathrm{pg} / \mathrm{mL}$. C-terminal FGF23 was measured using second generation human FGF23 ELISA kit (Immutopics, San Clemente, California, USA) according to the manufacturer's protocol. The intra-assay and inter-assay coefficients of variation as reported by the manufacturer were 1.4-2.4\% (FGF23 levels 33.7-302 RU/mL) and 2.4-4.7\% (FGF23 levels 33.6-293 $\mathrm{RU} / \mathrm{mL})$, respectively.

\section{Cardiovascular parameters}

Cardiovascular parameters were evaluated using left ventricular mass index (LVMI) and pulse wave velocity (PWV), which represent left ventricular hypertrophy and arterial stiffness, respectively. Two-dimensional echocardiography was conducted by experts at each hospital, and the LVMI was calculated by dividing the left ventricular (LV) mass by theheight ${ }^{2.7}$ [22]. LVMI was calculated in the cohort center using the same formula. LV mass was calculated by the formula $0.8 \times\left\{1.04 \times\left[\left(\text { LVIDd }^{2} \text { PWTd }+ \text { SWTd }\right)^{3}-\right.\right.$ LVIDd $\left.\left.^{3}\right]\right\}+$ 0.6 (g), where LVIDd, PWTd, and SWTd are LV internal diameter at end diastole, posterior wall thickness at end diastole, and septal wall thickness at end diastole, respectively [23]. Left ventricular hypertrophy (LVH) was defined 
as LVMI $\geq 50 \mathrm{~g} / \mathrm{m}^{2.7}$ in men and $\geq 47 \mathrm{~g} / \mathrm{m}^{2.7}$ in women [24]. Relative wall thickness (RWT) was calculated to as two times posterior wall thickness/LV internal linear dimension in diastole (RWT $=[2 \mathrm{x}$ PWTd $] / \mathrm{LVIDd})$. RWT was considered to be increased if $>0.42$. LVMI and RWT were used to categorize LV geometry: normal (normal LVMI and normal RWT), concentric remodeling (normal LVMI and RWT > 0.42), concentric LVH (increased LVMI and RWT > 0.42 ), eccentric LVH (increased LVMI and RWT $\leq 0.42$ ). Systolic heart dysfunction and diastolic heart dysfunction were defined as an LV ejection fraction $<50 \%$ and a ratio (E/E' ratio) of mitral peak velocity of early filling (E) to early diastolic mitral annular velocity $\left(E^{\prime}\right)>15$ on echocardiography, respectively $[25,26]$.Arterial stiffness was measured with brachial-to-ankle PWV (baPWV) and heart-tofemoral PWV (hfPWV) [27]. Among the nine participating centers, hfPWV was measured only at five centers where the equipment was available. hfPWV represented central arterial stiffness, while baPWV represented peripheral arterial stiffness. The abdominal aorta calcification(AAC) score was measured with simple lateral lumbar radiography with a range of 0 to 24 [28]. The coronary artery calcium score (CACS) was measured by computed tomography and was presented as the Agatson score $[29,30]$.

\section{Statistical analyses}

Categorical variables were evaluated using the Chi-square test and presented as frequencies and percentages. Continuous variables were analyzed with one-way analysis of variance (ANOVA) or the Kruskal-Wallis test. The Kolmogorov-Smirnov test was used to analyze the normality of the distribution of parameters. Results are presented as the mean \pm standard deviation (SD) for normally distributed variables and the median (interquartile range [IQR]) for variables with skewed distribution. Participants were classified into quartiles according to their serum klotho level. We analyzed the serum klotho value across the CKD stages by Kruskall-Wallis test and $P$ value is the difference between CKD stages. $P$-for trend of klotho level with advanced CKD stages was measured by Jonckheere-Terpstra test (Jonckheere trend test). We analyzed LVMI and PWV across the klotho quartiles by ANOVA and $P$ value is the difference between klotho quartiles. $P$-for trend of LMVI and PWV value with higher klotho quartiles was measured using ANOVA trend analyses using polynomial contrasts. We employed a multivariable linear regression model analysis with adjustment (enter method) to investigate the associations of left ventricular hypertrophy and PWV with serum klotho level. LVMI and PWV are continuous variables. LMVI and PWV (dependent variables) corresponding to the independent variables fulfilled the assumptions for linear regression model: normality, homoscedasticity, independence, and linearity. For all linear regression models, variables were selected based on the prior studies and physiological reasoning [31-33]. Collinearity among variables was tested. $P$-values $<0.05$ were considered statistically significant. The SPSS statistical software (SPSS version 18.0, Chicago, IL, USA) was used for all descriptive and outcome analyses.

\section{Results \\ Demographic and baseline clinical characteristics of participants}

The clinical characteristics of the study patients at enrollment are shown in Table 1. Among 2101 patients, the mean age was $53.6 \pm 12.2$ years, and $61.1 \%$ were male. The mean eGFR was $53.0 \pm 30.7 \mathrm{~mL} / \mathrm{min} / \mathrm{m}^{2}$. Patients with diabetes mellitus (DM) and hypertension (HTN) comprised $33.9 \%$ and $96.1 \%$ of the subjects, respectively. The median serum klotho level was 536 (interquartile range [IQR]:420-667) pg/mL. Serum klotho levels according to CKD stages are shown in Fig. 1. Advanced CKD stages were associated with lower serum klotho levels $(P<0.001$, $P$ for linear trend $<0.001)$.

\section{Comparison of baseline characteristics according to serum klotho levels}

We compared baseline characteristics classified by the klotho quartiles (Table 1). Patients in the highest (4th) quartile group were younger and had a lower body mass index (BMI). eGFR and hemoglobin levels were higher in the 4th quartile klotho group. Uric acid, C-reactive protein (CRP), phosphorus, and intact parathyroid hormone(iPTH) were lower in the 4th quartile klotho group.

\section{Serum klotho and cardiovascular parameters}

Ascending quartiles of klotho were significantly associated with decreased LMVI $(P<0.001, P$ for linear trend $<0.001$; Fig. 2). A total of 509 (24.8\%) patients had LVH (281 [22.4\%] in male, 228 [28.6\%] in female). LV geometry was not significantly different among the klotho quartile groups $(P=0.084)$. Table 2 summarizes the results of the multivariable linear regression analysis of the association between klotho and LVMI. After adjustment for age, gender, DM, HTN, BMI, systolic blood pressure, eGFR, hemoglobin, phosphorus, corrected calcium, and FGF23, serum klotho had a significant inverse association with LVMI $(\beta-0.04$; 95\% CI -0.004, $-0.00007 ; P=0.041$; Table 2). baPWV had a tendency of decreasing across the quartiles of klotho but it is not statistically significant $(P=0.127$; Fig. $3 \mathrm{~A})$.Ascending quartiles of klotho were significantly associated with decreased hfPWV $(P=0.022, P$ for linear trend $=0.002$; Fig. 3B). However, there was no significant association of serum klotho with baPWV $(\beta$ 0.003; 95\% CI -0.04, 0.05; $P=0.876)$ and hfPWV $(\beta-0.013 ; 95 \%$ CI $-0.06,0.034 ; P=$ 0.564 ) after adjustment (Table 3 ). AAC and CACS values were not significantly different among the klotho groups (Table 1). Systolic or diastolic heart function values as 
Table 1 The clinical characteristics of the study subjects at enrollment stratified by serum klotho level

\begin{tabular}{|c|c|c|c|c|c|c|c|}
\hline \multirow[t]{2}{*}{ Characteristics } & \multirow{2}{*}{$\begin{array}{l}\text { Total } \\
(N=2101)\end{array}$} & \multicolumn{4}{|l|}{ Klotho groups } & \multirow[t]{2}{*}{$P$-value } & \multirow[t]{2}{*}{$P$ for trend } \\
\hline & & $\begin{array}{l}\text { 1st quartile } \\
(n=524) \\
(99-419 \mathrm{pg} / \mathrm{mL})\end{array}$ & $\begin{array}{l}\text { 2nd quartile } \\
(n=528) \\
(420-536 \mathrm{pg} / \mathrm{mL})\end{array}$ & $\begin{array}{l}\text { 3rd quartile } \\
(n=523) \\
(537-666 \mathrm{pg} / \mathrm{mL})\end{array}$ & $\begin{array}{l}\text { 4th quartile } \\
(n=526) \\
(667-3641 \mathrm{pg} / \mathrm{mL})\end{array}$ & & \\
\hline Age (mean $\pm S D)$ & $53.6 \pm 12.2$ & $54.0 \pm 11.8$ & $54.6 \pm 11.9$ & $53.5 \pm 12.2$ & $52.2 \pm 12.5$ & 0.015 & 0.007 \\
\hline Gender, male, n (\%) & $1284(61.1)$ & $330(63.0)$ & $319(60.4)$ & $317(60.6)$ & $318(60.5)$ & 0.795 & 0.440 \\
\hline BMI $\left(\mathrm{kg} / \mathrm{m}^{2}\right)$ & $24.5 \pm 3.4$ & $24.8 \pm 3.5$ & $24.6 \pm 3.2$ & $24.5 \pm 3.4$ & $24.2 \pm 3.3$ & 0.036 & 0.005 \\
\hline $\mathrm{DM}, \mathrm{n}(\%)$ & $712(33.9)$ & $171(32.6)$ & $192(36.4)$ & $172(32.9)$ & $177(33.7)$ & 0.560 & 0.962 \\
\hline HTN, n (\%) & $2020(96.1)$ & $507(96.8)$ & 517 (97.9) & $501(95.8)$ & $495(94.1)$ & 0.011 & 0.007 \\
\hline SBP $(\mathrm{mmHg})$ & $128.5 \pm 16.4$ & $129.3 \pm 16.1$ & $128.5 \pm 16.6$ & $127.9 \pm 16.9$ & $128.5 \pm 16.1$ & 0.599 & 0.370 \\
\hline DBP (mmHg) & $76.9 \pm 11.2$ & $76.7 \pm 11.8$ & $77.0 \pm 11.1$ & $76.6 \pm 11.2$ & $77.2 \pm 10.5$ & 0.786 & 0.667 \\
\hline ACEi or ARB, yes, n (\%) & 1795 (85.6) & $458(87.4)$ & $461(87.5)$ & $442(84.7)$ & $434(82.7)$ & 0.075 & 0.013 \\
\hline $\begin{array}{l}\text { eGFR } \\
\left(\mathrm{ml} / \mathrm{min} / 1.73 \mathrm{~m}^{2}\right)\end{array}$ & $53.0 \pm 30.7$ & $48.2 \pm 28.6$ & $47.6 \pm 27.8$ & $54.6 \pm 31.6$ & $61.7 \pm 32.5$ & $<0.001$ & $<0.001$ \\
\hline Hemoglobin (g/dL) & $12.8 \pm 2.0$ & $12.6 \pm 1.9$ & $12.6 \pm 2.0$ & $12.9 \pm 2.0$ & $13.3 \pm 2.1$ & 0.001 & $<0.001$ \\
\hline Uric acid (mg/dL) & $7.0 \pm 1.9$ & $7.4 \pm 2.0$ & $7.2 \pm 1.9$ & $6.9 \pm 1.9$ & $6.6 \pm 1.8$ & $<0.001$ & $<0.001$ \\
\hline Albumin (g/dL) & $4.2 \pm 0.4$ & $4.1 \pm 0.4$ & $4.2 \pm 0.4$ & $4.2 \pm 0.4$ & $4.2 \pm 0.5$ & 0.316 & 0.068 \\
\hline Total cholesterol (mg/dL) & $174.3 \pm 39.4$ & $174.0 \pm 39.3$ & $172.0 \pm 38.0$ & $172.5 \pm 40.3$ & $178.8 \pm 39.6$ & 0.021 & 0.053 \\
\hline $\begin{array}{l}\text { CRP, median, } \\
(\mathrm{Q} 1, \mathrm{Q} 3)(\mathrm{mg} / \mathrm{dL})\end{array}$ & $0.06(0.02,0.17)$ & $0.08(0.03,0.20)$ & $0.06(0.03,0.16)$ & $0.06(0.02,0.16)$ & $0.05(0.02,0.13)$ & $<0.001$ & $<0.001$ \\
\hline Phosphorus (mg/dL) & $3.7 \pm 0.7$ & $3.8 \pm 0.7$ & $3.7 \pm 0.7$ & $3.7 \pm 0.7$ & $3.6 \pm 0.6$ & 0.004 & 0.001 \\
\hline Corrected Ca (mg/dL) & $9.0 \pm 0.4$ & $9.0 \pm 0.4$ & $9.0 \pm 0.5$ & $9.0 \pm 0.4$ & $8.9 \pm 0.4$ & 0.505 & 0.530 \\
\hline $\begin{array}{l}\text { Klotho, median } \\
(\mathrm{Q} 1, \mathrm{Q} 3)(\mathrm{pg} / \mathrm{mL})\end{array}$ & $536(420,667)$ & $335(269,383)$ & $479(449,505)$ & $593(562,626)$ & $788(714,913)$ & $<0.001$ & $<0.001$ \\
\hline $\begin{array}{l}\text { 25(OH)VitD, median } \\
\text { (Q1, Q3) (ng/mL) }\end{array}$ & $16.5(12.7,21.3)$ & $16.7(13.5,21.7)$ & $16.6(13.1,21.2)$ & $16.4(12.6,21.3)$ & $16.1(12.1,21.0)$ & 0.040 & 0.005 \\
\hline $\begin{array}{l}\text { 1,25(OH) })_{2} \text { VitD, median } \\
(\mathrm{Q} 1, \mathrm{Q} 3)(\mathrm{pg} / \mathrm{mL})\end{array}$ & $25.4(20.1,33.7)$ & $26.4(20.1,36.2)$ & $24.6(19.1,31.6)$ & $24.3(19.4,32.5)$ & $26.6(21.4,34.4)$ & $<0.001$ & 0.599 \\
\hline $\begin{array}{l}\text { iPTH, median } \\
(\mathrm{Q} 1, \mathrm{Q} 3)(\mathrm{pg} / \mathrm{mL})\end{array}$ & $51.5(33.2,84.0)$ & $55.0(35.0,86.1)$ & $53.0(34.5,88.8)$ & $52.6(33.9,86.8)$ & $46.3(30.2,74.8)$ & 0.005 & 0.003 \\
\hline $\begin{array}{l}\text { C-terminal FGF23, } \\
\text { median (Q1, Q3) } \\
\text { (RU/mL) }\end{array}$ & $17.9(0.4,31.3)$ & $18.5(0.3,30.2)$ & $19.0(1.0,32.3)$ & $18.6(0.8,31.5)$ & $10.4(0.2,31.4)$ & 0.094 & 0.419 \\
\hline $\begin{array}{l}\text { UACR, }>300 \\
\mathrm{mg} / \mathrm{g}, \mathrm{n}(\%)\end{array}$ & $884(49.9)$ & $217(52.3)$ & $255(56.0)$ & $205(45.5)$ & $207(45.9)$ & $<0.001$ & $<0.001$ \\
\hline $\begin{array}{l}\text { LVMI, median } \\
(\mathrm{Q} 1, \mathrm{Q} 3)\left(\mathrm{g} / \mathrm{m}^{2.7}\right)\end{array}$ & $40.3(33.8,48.6)$ & $41.3(34.7,50.2)$ & $40.9(34.4,49.2)$ & $39.5(33.5,49.3)$ & $39.6(32.5,46.7)$ & $<0.001$ & $<0.001$ \\
\hline LVH, n (\%) & $509(24.8)$ & $145(28.3)$ & $139(27.0)$ & $128(24.8)$ & $97(19.2)$ & 0.004 & 0.001 \\
\hline LV geometry, n (\%) & & & & & & 0.084 & 0.006 \\
\hline Normal & $1248(60.9)$ & $303(59.1)$ & $304(59.0)$ & $316(61.2)$ & $325(64.2)$ & & \\
\hline $\begin{array}{l}\text { Concentric } \\
\text { remodeling }\end{array}$ & $293(14.3)$ & $65(12.7)$ & $72(14.0)$ & $72(14.0)$ & $84(16.6)$ & & \\
\hline Eccentric LVH & $254(12.4)$ & $76(14.8)$ & 70 (13.6) & $60(11.6)$ & $48(9.5)$ & & \\
\hline Concentric LVH & $255(12.4)$ & 69 (13.5) & $69(13.4)$ & $68(13.2)$ & $49(9.7)$ & & \\
\hline baPWV (cm/s) & $1534 \pm 344$ & $1552 \pm 348$ & $1552 \pm 322$ & $1527 \pm 357$ & $1507 \pm 347$ & 0.127 & 0.024 \\
\hline${ }^{a} \mathrm{hfPWV}(\mathrm{cm} / \mathrm{s})$ & $1018 \pm 274$ & $1053 \pm 300$ & $1028 \pm 257$ & $1005 \pm 277$ & $989 \pm 258$ & 0.022 & 0.002 \\
\hline$A A C \geq 1, n(\%)$ & $703(35.0)$ & $172(35.0)$ & $194(38.0)$ & $183(36.6)$ & $154(30.6)$ & 0.079 & 0.127 \\
\hline
\end{tabular}


Table 1 The clinical characteristics of the study subjects at enrollment stratified by serum klotho level (Continued)

\begin{tabular}{|c|c|c|c|c|c|c|c|}
\hline \multirow[t]{2}{*}{ Characteristics } & \multirow{2}{*}{$\begin{array}{l}\text { Total } \\
(N=2101)\end{array}$} & \multicolumn{4}{|l|}{ Klotho groups } & \multirow[t]{2}{*}{$P$-value } & \multirow[t]{2}{*}{$P$ for trenc } \\
\hline & & $\begin{array}{l}\text { 1st quartile } \\
(n=524) \\
(99-419 \mathrm{pg} / \mathrm{mL}) \\
\end{array}$ & $\begin{array}{l}\text { 2nd quartile } \\
(n=528) \\
(420-536 \mathrm{pg} / \mathrm{mL})\end{array}$ & $\begin{array}{l}\text { 3rd quartile } \\
(n=523) \\
(537-666 \mathrm{pg} / \mathrm{mL}) \\
\end{array}$ & $\begin{array}{l}\text { 4th quartile } \\
(n=526) \\
(667-3641 \mathrm{pg} / \mathrm{mL})\end{array}$ & & \\
\hline CACS $>100, n(\%)$ & $498(24.6)$ & $133(26.2)$ & $131(25.7)$ & $121(24.0)$ & $113(22.6)$ & 0.528 & 0.143 \\
\hline LVEF $<50 \%$ & $30(1.5)$ & $8(1.5)$ & $5(1.0)$ & $7(1.3)$ & $10(2.0)$ & 0.607 & 0.497 \\
\hline$E / E^{\prime}>15$ & $176(8.6)$ & $46(9.0)$ & $47(9.1)$ & $42(8.2)$ & $41(8.1)$ & 0.923 & 0.536 \\
\hline
\end{tabular}

${ }^{\mathrm{a}} 1243$ patients measured hfPWV (vs. 1907 patients measured baPWV) at the study enrollment

$\mathrm{SD}$, standard deviation; BMI, body mass index; DM, diabetes mellitus; HTN, hypertension; SBP, systolic blood pressure; DBP, diastolic blood pressure; eGFR, estimated glomerular filtration rate by CKD-EPI creatinine equation; CRP, C-reactive protein; Ca, calcium; $25(\mathrm{OH}) \mathrm{VitD}, 25-\mathrm{hydroxy}$ vitamin $\mathrm{D} ; 1,25(\mathrm{OH})_{2}$ VitD, 1,25-hydroxy vitamin D; iPTH, intact parathyroid hormone; FGF23, fibroblast growth factor 23; UACR, random urine albumin-creatinine ratio; ACEi, angiotensin converting enzyme inhibitor; ARB, angiotensin receptor blocker; LVMI, left ventricular mass index; LVH, left ventricular hypertrophy; baPWV, brachial-to-ankle pulse wave velocity; hfPWV, heart-to-femoral pulse wave velocity; AAC, Abdominal aorta calcification; CACS, coronary artery calcium score; LVEF, left ventricular ejection fraction; $E / E^{\prime}$, ratio of mitral peak velocity of early filling $(E)$ to early diastolic mitral annular velocity $\left(E^{\prime}\right)$

LVEF and E/E' were not significantly different among the klotho groups (Table 1).

\section{Discussion}

The kidney is the principal organ for production of klotho, and CKD is known to be associated with a klothodeficiency state. CKD patients suffer from a high burden of CVD. In the present study, the serum klotho level was lower in advanced CKD stages. Klotho exhibited an independent negative association with LVMI. However, there was no significant association between klotho and PWV after adjustment in our subjects. Abdominal aorta calcification and coronary artery calcification were not significantly different among the klotho quartile groups. No differences in systolic or diastolic heart dysfunction were observed across klotho quartiles.

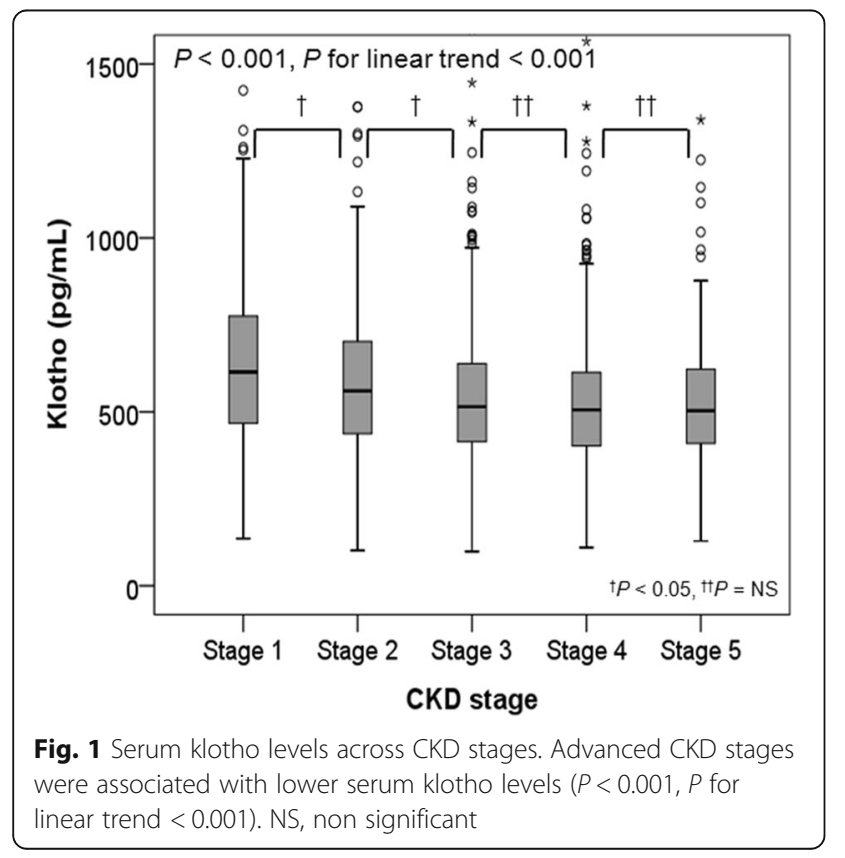

Previous studies showed that CKD patients were more likely to have cardiac structural changes in the absence of decreased LV ejection fraction [34, 35]. Lower prevalence of systolic and diastolic heart dysfunction was not surprising, given the exclusion of subjects with severe heart failure (New York Heart Association Class III or IV) from enrollment in the present study.

Xieet al. [12] showed that klotho-deficient CKD mice have aggravated cardiac hypertrophy and cardiac fibrosis compared with wild-type CKD mice. Intravenous delivery of a transgene encoding soluble klotho attenuated cardiac hypertrophy in the klotho-deficient CKD mice. The authors explained that downregulation of the stress-induced transient receptor potential canonical 6 (TRPC6)-mediated gene amplification loop by soluble klotho may play a role in the cardioprotection of uremic hearts [36].Yang et al.[16]reported that klotho protects against indoxyl sulphate-induced cardiac hypertrophy in CKD mice. They also showed that serum

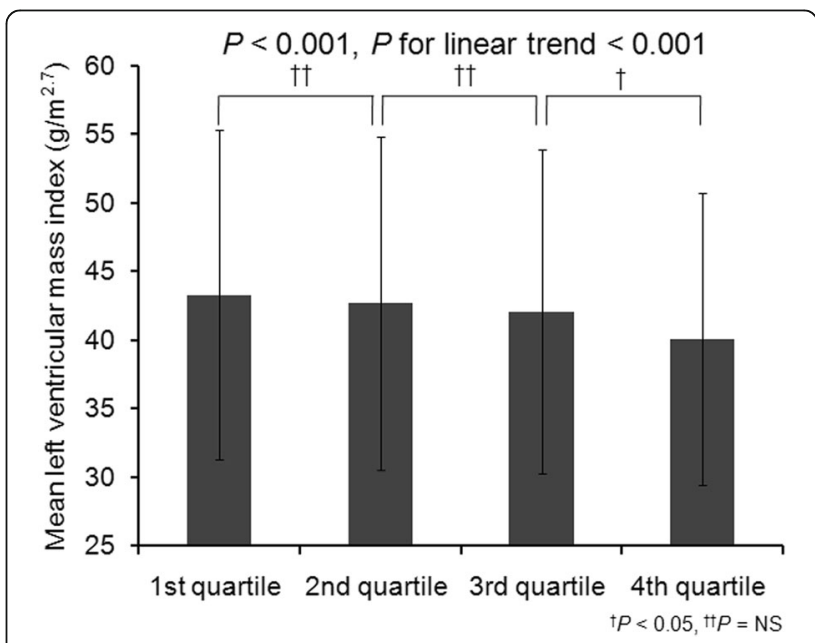

Fig. 2 Mean left ventricular mass index across the quartiles of klotho. Ascending quartiles of klotho were significantly associated with decreased left ventricular mass index $(P<0.001, P$ for linear trend <0.001). NS, non significant 
Table 2 Multivariable linear regression analysis presenting associations between klotho and left ventricular mass index

\begin{tabular}{|c|c|c|c|c|c|c|}
\hline \multirow[t]{2}{*}{ Variable } & \multicolumn{2}{|l|}{ Model A } & \multicolumn{2}{|l|}{ Model B } & \multicolumn{2}{|l|}{ Model C } \\
\hline & $\beta(95 \% \mathrm{Cl})$ & $P$-value & $\beta(95 \% \mathrm{Cl})$ & $P$-value & $\beta(95 \% \mathrm{Cl})$ & $P$-value \\
\hline Klotho $(\mathrm{pg} / \mathrm{mL})$ & $-0.09(-0.006,-0.002)$ & $<0.001$ & $-0.079(-0.005,-0.002)$ & $<0.001$ & $-0.04(-0.004,-0.00007)$ & 0.041 \\
\hline
\end{tabular}

Model A: Adjusted for klotho, age and gender

Model B: Adjusted for klotho, age, gender, DM, HTN, BMI, and SBP

Model C: Adjusted for klotho, age, gender, DM, HTN, BMI, SBP, eGFR, hemoblogin, phosphorus, corrected Ca, and FGF23

$\beta$, Standardized coefficient; $\mathrm{Cl}$, confidence interval; DM, diabetes mellitus; HTN, hypertension; BMI, body mass index; SBP, systolic blood pressure; eGFR, estimated glomerular filtration rate by CKD-EPI creatinine equation; Ca, calcium; FGF23, fibroblast growth factor 23

klotho levels are associated with the development of LVH in patients with CKD. They did not show an association between klotho and LVMI for CKD patients in an adjustment model. Most animal study results have suggested that klotho deficiency is associated with cardiac hypertrophy. However, clinical studies have shown mixed results with regards to serum klotho and cardiac hypertrophy. Tanaka et al. [37] reported that the lowest klotho tertile was associated with LV hypertrophy and systolic dysfunction only among patients with CKD stage G3a and G3b, respectively. Buiten et al. [17] showed that serum klotho was not independently associated with CVD, including LVMI, among 127 dialysis patients. This study was conducted with a small number of dialysis patients compared to our study. They also described that the association of soluble klotho with cardiovascular parameters might be diminished since the patients already developed end stage renal disease [17]. Seiler et al. [38] showed that soluble klotho was not significantly associated with cardiovascular outcomes for 444 patients with CKD stage 2-4. They did not assess each of cardiovascular parameters and mean eGFR was lower than that of our study subjects ( $45 \pm 16$ vs. $53.0 \pm 30.7 \mathrm{ml} /$ $\min / 1.73 \mathrm{~m}^{2}$ ). Our study included all stages of CKD patients and presented that klotho could be a marker of LVMI in predialysis CKD patients. The reason for the discrepancies between those studies and ours remains uncertain. However, there are possible explanations. First, these studies differed in race, kidney function, and number of subjects. Second, we examined cardiovascular parameters, rather than cardiovascular outcomes. Thirdly, patients with severe heart failure (New York Heart Association Class III or IV) were excluded in our study. However, the present study has a much greater statistical power, due to a larger number of CKD subjects analyzed. Soluble klotho plays important roles in antiaging, anti-oxidant, and anti-vascular calcification [39],and CKD as a klotho-deficient state may have a close association with chronic cardiovascular complications. The present study showed the association of klotho with LVMI in a large number of CKD patients, with adjustment for markers of mineral bone metabolism such as phosphorous and calcium.

In an experimental study, klotho gene delivery into skeletal muscle inhibited medial hypertrophy of the aorta in an animal model of atherosclerotic disease [40], and klotho deficiency-induced arterial stiffening was mediated by upregulation of aldosterone levels [14]. Soluble klotho protects endothelial integrity by regulating calcium entry into vascular endothelial cells [40, 41]. Kitagawa et al. [42] reported that the serum klotho level was a significant determinant of arterial stiffness, defined as baPWV $\geq 1400 \mathrm{~cm} / \mathrm{s}$ in 114 CKD patients. They showed a significant association only at baPWV $\geq 1400 \mathrm{~cm} / \mathrm{s}$. In our study, we also analyzed hfPWV as a central arterial stiffness marker. In another clinical study, arterial stiffness measured by baPWV increased in 109 CKD patients, but it was not related to klotho [43]. This study was performed only with a small number of diabetic CKD patients. Thus, there have been discrepancies among clinical study results. Further studies
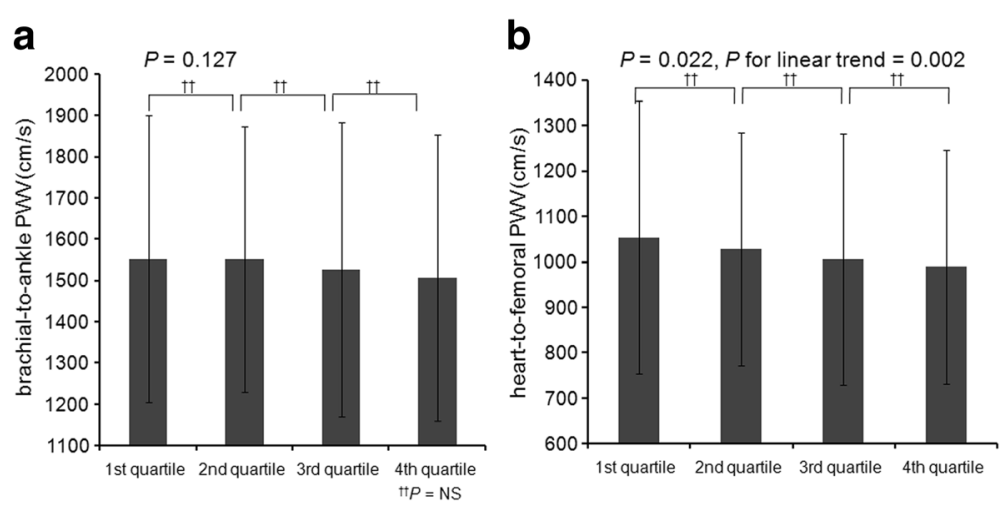

Fig. 3 Mean pulse wave velocity across the quartiles of klotho. a Brachial-to-ankle pulse wave velocity had a tendency of decreasing across the quartiles of klotho. $\mathbf{b}$ Ascending quartiles of klotho were significantly associated with decreased heart-to-femoral $P W V(P=0.022, P$ for linear trend $=0.002$ ). PW, pulse wave velocity; NS, non significant 
Table 3 Multivariable linear regression analysis presenting associations between klotho and pulse wave velocity

\begin{tabular}{|c|c|c|c|c|c|c|c|}
\hline \multirow[t]{2}{*}{ 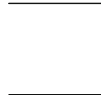 } & \multirow[t]{2}{*}{ Variable } & \multicolumn{2}{|l|}{ Model A } & \multicolumn{2}{|l|}{ Model B } & \multicolumn{2}{|l|}{ Model C } \\
\hline & & $\overline{\beta(95 \% \mathrm{Cl})}$ & $P$-value & $\overline{\beta(95 \% \mathrm{Cl})}$ & $P$-value & $\overline{\beta(95 \% \mathrm{Cl})}$ & $P$-value \\
\hline baPWV & Klotho (pg/mL) & $-0.011(-0.07,0.04)$ & 0.582 & $-0.017(-0.07,0.02)$ & 0.329 & $0.003(-0.04,0.05)$ & 0.876 \\
\hline hfPWV & Klotho (pg/mL) & $-0.029(-0.09,0.02)$ & 0.228 & $-0.037(-0.09,0.01)$ & 0.087 & $-0.013(-0.06,0.034)$ & 0.564 \\
\hline
\end{tabular}

Model A: Adjusted for klotho, age and gender

Model B: Adjusted for klotho, age, gender, DM, HTN, BMI, and SBP

Model C: Adjusted forklotho, age, gender, DM, HTN, BMI, SBP, eGFR, hemoblogin, phosphorus, corrected Ca, and FGF23

$\beta$ Standardized coefficient, $C I$ confidence interval, baPWV brachial-to-ankle pulse wave velocity; $h f P W V$, heart-to-femoral pulse wave velocity; $D M$ diabetes mellitus, HTN hypertension, BMI body mass index, SBP systolic blood pressure, eGFR estimated glomerular filtration rate by CKD-EPI creatinine equation, Ca calcium, FGF23 fibroblast growth factor 23

are needed to elucidate the association between klotho and arterial stiffness in CKD patients. This study has several limitations. First, owing to the cross-sectional nature of the study, it is hard to demonstrate the cause-effect inferences about the relationship between serum klotho levels and cardiac hypertrophy or arterial stiffness. Second, serum klotho has a circadian variation, meaning that examination at a fixed time is necessary [44]. Thirdly, we measured cterminal FGF23 in this study. Lack of agreement between c-terminal and intact FGF23 measurements and also differences in their association with other biochemical parameters have been reported [45]. However, both the higher cterminal and intact FGF23 values have been associated with increased mortality and poor outcomes in CKD patients.

\section{Conclusions}

Serum klotho was an independent biomarker of LVMI but not arterial stiffness and vascular calcification. Further studies are warranted to elucidate the clinico-pathogenic significance of klotho for cardiovascular parameters, and whether any interventions to maintain or increase the serum klotho level can prevent cardiovascular events and mortality in CKD patients.

\begin{abstract}
Abbreviations
AAC: abdominal aorta calcification; ANOVA: one-way analysis of variance; BMI: body mass index; CACS: coronary artery calcium score; Cl: confidence interval; CKD: chronic kidney disease; CKD-EPI: Chronic Kidney Disease Epidemiology Collaboration; CRP: C-reactive protein; CVD: cardiovascular disease; DM: diabetes mellitus; eGFR: estimated glomerular filtration rate; ELISA: enzyme-linked immunosorbent assay; HTN: hypertension; IDMS: isotope dilution mass spectrometry; iPTH: intact parathyroid hormone; IQR: interquartile range; KNOWCKD: KoreaN Cohort Study for Outcome in Patients With Chronic Kidney Disease; LV: left ventricular; LVH: left ventricular hypertrophy; LVIDd: left ventricular internal diameter at end diastole; LVMI: left ventricular mass index; PWTd: posterior wall thickness at end diastole; PWV: pulse wave velocity; RW: relative wall thickness;

SD: standard deviation; SWTd: septal wall thickness at end diastole; VitD: vitamin D
\end{abstract}

\section{Acknowledgments}

The authors gratefully thank to the clinical research staff and nurses of KNOW-CKD study.

\section{Funding}

This study was supported by the research program funded by the Korea Center for Disease Control and Prevention (2011E3300300, 2012E3301100, 2013E3301600, 2013E3301601, 2013E3301602, and 2016E3300200).
Availability of data and materials

The data supporting the findings can be obtained on request to the corresponding author.

\section{Authors' contributions}

Research idea and study design: HJK, DWC, KHO, CA; data acquisition: HJK, EK, $\mathrm{KHO}$; data analysis/interpretation: HJK, DWC, KHO, YKO, YHK, SHH, THY; statistical analysis: HJK, JL, KHO; supervision or mentorship: KHO, CA. Each author contributed important intellectual content during manuscript drafting and accepts accountability for the overall work by ensuring that questions pertaining to the accuracy or integrity of any portion of the work are appropriately investigated and resolved. $\mathrm{KHO}$ takes responsibility that this study has been reported honestly, accurately, and transparently; that no important aspects of the study have been omitted; and that any discrepancies from the study as planned have been explained. All authors read and approved the final manuscript.

\section{Ethics approval and consent to participate}

The study protocol was approved by the ethical committee of each participating clinical center, including the Institutional Review Boards of Seoul National University Hospital, Severance Hospital, Kangbuk Samsung Medical Center, Seoul St. Mary's Hospital, Gil Hospital, Eulji General Hospital, Chonnam National University Hospital, and Busan Paik Hospital. All participating patients provided written informed consent. The study protocol was in accordance with the principles of the Declaration of Helsinki.

\section{Consent for publication}

Not applicable.

\section{Competing interest}

The authors declare that they have no competing interests.

\section{Publisher's Note}

Springer Nature remains neutral with regard to jurisdictional claims in published maps and institutional affiliations.

\section{Author details}

${ }^{1}$ Department of Internal Medicine, Dongguk University College of Medicine, Gyeongju-si, Gyeongsangbuk-do, South Korea. ${ }^{2}$ Department of Internal Medicine, Seoul National University College of Medicine, Seoul, Korea. ${ }^{3}$ Department of Internal Medicine, Inje University, Busan Paik Hospital, Busan, South Korea. ${ }^{4}$ Department of Internal Medicine, Yonsei University College of Medicine, Seoul, South Korea. ${ }^{5}$ Medical Research Collaborating Center, Seoul National University Hospital and Seoul, National University College of Medicine, Seoul, South Korea.

Received: 2 September 2017 Accepted: 20 February 2018

Published online: 05 March 2018

\section{References}

1. Foley RN, Parfrey PS, Sarnak MJ. Clinical epidemiology of cardiovascular disease in chronic renal disease. Am J Kidney Dis. 1998;32:S112-9.

2. Go AS, Chertow GM, Fan D, McCulloch CE, Hsu CY. Chronic kidney disease and the risks of death, cardiovascular events, and hospitalization. N Engl J Med. 2004;351:1296-305. 
3. Taddei S, Nami R, Bruno RM, Quatrini I, Nuti R. Hypertension, left ventricular hypertrophy and chronic kidney disease. Heart Fail Rev. 2011;16:615-20.

4. Middleton RJ, Parfrey PS, Foley RN. Left ventricular hypertrophy in the renal patient. J Am Soc Nephrol. 2001;12:1079-84.

5. Gross ML, Hypertrophy RE. Fibrosis in the cardiomyopathy of uremiabeyond coronary heart disease. Semin Dial. 2008:21:308-18.

6. Glassock RJ, Pecoits-Filho R, Barberato SH. Left ventricular mass in chronic kidney disease and ESRD. Clin J Am Soc Nephrol. 2009;4(Suppl 1):S79-91.

7. O'Rourke M. Mechanical principles in arterial disease. Hypertension. 1995;26:2-9.

8. Temmar M, Liabeuf S, Renard C, Czernichow S, Esper NE, Shahapuni I, et al. Pulse wave velocity and vascular calcification at different stages of chronic kidney disease. J Hypertens. 2010;28:163-9.

9. Townsend RR. Arterial stiffness and chronic kidney disease: lessons from the chronic renal insufficiency cohort study. Curr Opin Nephrol Hypertens. 2015; 24:47-53.

10. Kuro-o M, Matsumura $Y$, Aizawa H, Kawaguchi H, Suga T, Utsugi T, et al. Mutation of the mouse klotho gene leads to a syndrome resembling ageing. Nature. 1997;390:45-51

11. Hu MC, Kuro-o M, Moe OW. Klotho and chronic kidney disease. Contrib Nephrol. 2013;180:47-63

12. Xie J, Yoon J, An SW, Kuro-o M, Huang CL. Soluble klotho protects against uremic cardiomyopathy independently of fibroblast growth factor 23 and phosphate. J Am Soc Nephrol. 2015;26:1150-60.

13. Hu MC, Shi M, Zhang J, Quinones H, Griffith C, Kuro-o M, et al. Klotho deficiency causes vascular calcification in chronic kidney disease. J Am Soc Nephrol. 2011;22:124-36.

14. Chen K, Zhou X, Sun Z. Haplodeficiency of klotho gene causes arterial stiffening via upregulation of Scleraxis expression and induction of autophagy. Hypertension. 2015;66:1006-13.

15. Bae EHI. Klotho deficiency independently associated with cardiovascular risk in chronic kidney disease? Kidney Res Clin Pract. 2016;35:1-2.

16. Yang K, Wang C, Nie L, Zhao X, Gu J, Guan X, et al. Klotho protects against Indoxyl Sulphate-induced myocardial hypertrophy. J Am Soc Nephrol. 2015; 26:2434-46.

17. Buiten MS, de Bie MK, Bouma-de Krijger A, van Dam B, Dekker FW, Jukema JW, et al. Soluble klotho is not independently associated with cardiovascular disease in a population of dialysis patients. BMC Nephrol. 2014;15:197.

18. Oh KH, Park SK, Park HC, Chin HJ, Chae DW, Choi KH, et al. KNOW-CKD (KoreaN cohort study for outcome in patients with chronic kidney disease): design and methods. BMC Nephrol. 2014;15:80.

19. Siekmann L. Determination of creatinine in human serum by isotope dilution-mass spectrometry. Definitive methods in clinical chemistry, IV. J Clin Chem Clin Biochem. 1985;23:137-44.

20. Levey AS, Stevens LA, Schmid CH, Zhang YL, Castro AF 3rd, Feldman HI, et al. A new equation to estimate glomerular filtration rate. Ann Intern Med. 2009;150:604-12.

21. Yamazaki Y, Imura A, Urakawa I, Shimada T, Murakami J, Aono Y, et al. Establishment of sandwich ELISA for soluble alpha-klotho measurement: age-dependent change of soluble alpha-klotho levels in healthy subjects. Biochem Biophys Res Commun. 2010;398:513-8.

22. de Simone G, Daniels SR, Devereux RB, Meyer RA, Roman MJ, de Divitiis O, et al. Left ventricular mass and body size in normotensive children and adults: assessment of allometric relations and impact of overweight. J Am Coll Cardiol. 1992;20:1251-60.

23. Lang RM, Bierig M, Devereux RB, Flachskampf FA, Foster E, Pellikka PA, et al. Recommendations for chamber quantification: a report from the American Society of Echocardiography's guidelines and standards committee and the chamber quantification writing group, developed in conjunction with the European Association of Echocardiography, a branch of the European Society of Cardiology. J Am Soc Echocardiogr. 2005;18:1440-63.

24. de Simone G, Devereux RB, Daniels SR, Koren MJ, Meyer RA, Laragh JH. Effect of growth on variability of left ventricular mass: assessment of allometric signals in adults and children and their capacity to predict cardiovascular risk. J Am Coll Cardiol. 1995;25:1056-62.

25. Ponikowski P, Voors AA, Anker SD, Bueno H, Cleland JG, Coats AJ, et al. ESC guidelines for the diagnosis and treatment of acute and chronic heart failure. Rev Esp Cardiol (Engl Ed) 2016. 2016;69:1167.

26. Paulus WJ, Tschope C, Sanderson JE, Rusconi C, Flachskampf FA, Rademakers FE, et al. How to diagnose diastolic heart failure: a consensus statement on the diagnosis of heart failure with normal left ventricular ejection fraction by the heart failure and echocardiography associations of the European Society of Cardiology. Eur Heart J. 2007;28: 2539-50.

27. Avolio AP, Chen SG, Wang RP, Zhang CL, Li MF, O'Rourke MF. Effects of aging on changing arterial compliance and left ventricular load in a northern Chinese urban community. Circulation. 1983;68:50-8.

28. Kauppila LI, Polak JF, Cupples LA, Hannan MT, Kiel DP, Wilson PW. New indices to classify location, severity and progression of calcific lesions in the abdominal aorta: a 25-year follow-up study. Atherosclerosis. 1997; 132:245-50.

29. Agatston AS, Janowitz WR, Hildner FJ, Zusmer NR, Viamonte M Jr, Detrano R. Quantification of coronary artery calcium using ultrafast computed tomography. J Am Coll Cardiol. 1990;15:827-32.

30. Budoff MJ, Nasir K, McClelland RL, Detrano R, Wong N, Blumenthal RS, et al. Coronary calcium predicts events better with absolute calcium scores than age-sex-race/ethnicity percentiles: MESA (multi-ethnic study of atherosclerosis). J Am Coll Cardiol. 2009;53:345-52.

31. Menon V, Gul A, Sarnak MJ. Cardiovascular risk factors in chronic kidney disease. Kidney Int. 2005;68:1413-8.

32. Levin A, Singer J, Thompson CR, Ross H, Lewis M. Prevalent left ventricular hypertrophy in the predialysis population: identifying opportunities for intervention. Am J Kidney Dis. 1996;27:347-54.

33. Astor BC, Arnett DK, Brown A, Coresh J. Association of kidney function and hemoglobin with left ventricular morphology among African Americans: the atherosclerosis risk in communities (ARIC) study. Am J Kidney Dis. 2004;43: 836-45.

34. Edwards NC, Hirth A, Ferro CJ, Townend JN, Steeds RP. Subclinical abnormalities of left ventricular myocardial deformation in early-stage chronic kidney disease: the precursor of uremic cardiomyopathy? J Am Soc Echocardiogr. 2008;21:1293-8.

35. Devereux RB, Roman MJ, Liu JE, Welty TK, Lee ET, Rodeheffer R, et al. Congestive heart failure despite normal left ventricular systolic function in a population-based sample: the strong heart study. Am J Cardiol. 2000;86: 1090-6.

36. Xie J, Cha SK, An SW, Kuro OM, Birnbaumer L, Huang CL. Cardioprotection by klotho through downregulation of TRPC6 channels in the mouse heart. Nat Commun. 2012;3:1238.

37. Tanaka S, Fujita S, Kizawa S, Morita H, Ishizaka N. Association between FGF23, alpha-klotho, and cardiac abnormalities among patients with various chronic kidney disease stages. PLoS One. 2016;11:e0156860.

38. Seiler S, Rogacev KS, Roth HJ, Shafein P, Emrich I, Neuhaus S, et al. Associations of FGF-23 and sKlotho with cardiovascular outcomes among patients with CKD stages 2-4. Clin J Am Soc Nephrol. 2014;9:1049-58.

39. Hu MC, Kuro-o M, Moe OW. Secreted klotho and chronic kidney disease. Adv Exp Med Biol. 2012;728:126-57.

40. Saito $Y$, Nakamura T, Ohyama Y, Suzuki T, lida A, Shiraki-lida T, et al. In vivo klotho gene delivery protects against endothelial dysfunction in multiple risk factor syndrome. Biochem Biophys Res Commun. 2000;276:767-72.

41. Kusaba T, Okigaki M, Matui A, Murakami M, Ishikawa K, Kimura T, et al. Klotho is associated with VEGF receptor-2 and the transient receptor potential canonical-1 Ca2+ channel to maintain endothelial integrity. Proc Natl Acad Sci U S A. 2010;107:19308-13.

42. Kitagawa $M$, Sugiyama $H$, Morinaga $H$, Inoue $T$, Takiue $K$, Ogawa A, et al. A decreased level of serum soluble klotho is an independent biomarker associated with arterial stiffness in patients with chronic kidney disease PLoS One. 2013;8:e56695.

43. Inci A, Sari F, Olmaz R, Coban M, Dolu S, Sarikaya M, et al. Soluble klotho levels in diabetic nephropathy: relationship with arterial stiffness. Eur Rev Med Pharmacol Sci. 2016;20:3230-7.

44. Carpenter TO, Insogna KL, Zhang JH, Ellis B, Nieman S, Simpson C, et al. Circulating levels of soluble klotho and FGF23 in X-linked hypophosphatemia: circadian variance, effects of treatment, and relationship to parathyroid status. J Clin Endocrinol Metab. 2010;95:E352-7.

45. Smith ER, Cai MM, McMahon LP, Holt SG. Biological variability of plasma intact and C-terminal FGF23 measurements. J Clin Endocrinol Metab. 2012; 97:3357-65 